\title{
Tunneling current through a quantum dot array
}

David M.-T. Kuo

Department of Electronic Engineering, Cheng-Shiu Institute of Technology, Kaohsiung 833, Taiwan

G. Y. Guo a)

Department of Physics, National Taiwan University, Taipei 106, Taiwan

Yia-Chung Chang

Department of Physics and Materials Research Laboratory, University of Illinois at Urbana-Champaign, Urbana, Illinois 61801

(Received 24 April 2001; accepted for publication 2 October 2001)

The tunneling current through a quantum dot array (QDA) is studied theoretically. Strong electron correlation effect is taken into account in the QDA in which the quantum dots provide a strong three-dimensional confinement effect. A mixed Hubbard and Anderson model is used to simulate the system. It is found that Coulomb charging splits the band resulting from interdot coupling into two subbands. The tunneling current is thus influenced significantly by both Coulomb charging and interdot coupling. () 2001 American Institute of Physics. [DOI: 10.1063/1.1420775]

Transport properties of quantum dot nanostructures have been extensively studied both experimently ${ }^{1-5}$ and theoretically. ${ }^{6-8}$ It was shown that electron-electron interaction in single electron transistors (SETs) made of quantum dot tunneling junctions can lead to interesting effects such as the Kondo resonance, Coulomb blockade, and parity effect. ${ }^{1-5}$ A SET is composed of two leads (reservoirs) and one or more quantum dots (QDs), which can be a normal metal, superconductor, ferromagnetic material, or semiconductor. The behavior of the tunneling current is very sensitive to the properties of the QDs.

Apart from SET, the QD system has many potential applications in electronic devices, including quantum dot lasers and infrared detectors. ${ }^{9,10}$ In the application in electronic devices (e.g., infrared detectors), QDAs are modulated by an applied field. The structure of the device is shown schematically in Fig. 1. In the application of infrared detectors, minimizing the dark current is an important issue. Thermionic current and phonon assisted current can usually be ignored at low temperatures, ${ }^{11}$ and the dark current is mainly due to the tunneling current. Increasing the area density of QDs can enhance the intensity of photocurrent. However, the dark current is increased as well. To achieve high detectivity in infrared detectors, it is necessary to optimize the ratio of photocurrent to dark current. Thus, it is desirable to study how the tunneling current is influenced by the interdot coupling and electron-electron interaction.

We start with the following Hamiltonian to describe the system shown by a schematic diagram in Fig. 1, in which an InAs QDs array is embedded into AlGaAs barrier connected by GaAs leads.

${ }^{a)}$ Electronic mail: gyguo@phys.ntu.edu.tw

$$
\begin{aligned}
H= & \sum_{\mathbf{k}, \sigma} \epsilon_{\mathbf{k}} a_{\mathbf{k}, \sigma}^{\dagger} a_{\mathbf{k}, \sigma}+\sum_{\mathbf{p}, \sigma} \epsilon_{\mathbf{p}} b_{p, \sigma}^{\dagger} b_{\mathbf{p}, \sigma}+\sum_{\mathbf{k}, i, \sigma} V_{\mathbf{k}, i} a_{\mathbf{k}, \sigma}^{\dagger} d_{i, \sigma} \\
& +h . c+\sum_{\mathbf{p}, i, \sigma} V_{\mathbf{p}, i} b_{\mathbf{p}, \sigma}^{\dagger} d_{i, \sigma}+h . c+\sum_{i, \sigma} \varepsilon_{i} d_{i, \sigma}^{\dagger} d_{i, \sigma} \\
& +\sum_{i, j, \sigma} t_{i, j} d_{j, \sigma}^{\dagger} d_{i, \sigma}+\sum_{i, \sigma} U_{i} n_{i, \uparrow} n_{i,-\downarrow},
\end{aligned}
$$

where the first two terms describe the left and right lead, respectively, the third and fourth term describe the coupling between the quantum dots array and the two leads, the last three terms describe electrons moving in the quantum dots array where only the nearest neighbor electron hopping integrals between QDs are included. Only one bound state (ground state) of QDs is taken into account due to the large energy spacing $\Delta E$ between the ground state and first excited state compared to temperature $T$.

Electron correlation in the two leads is ignored for Fermi liquid behavior (free electrons model). To simplify the problem we assume that all dots in the array are identical and consider only onsite Coulomb potential $U_{i}=U \approx 10 \mathrm{meV}$,

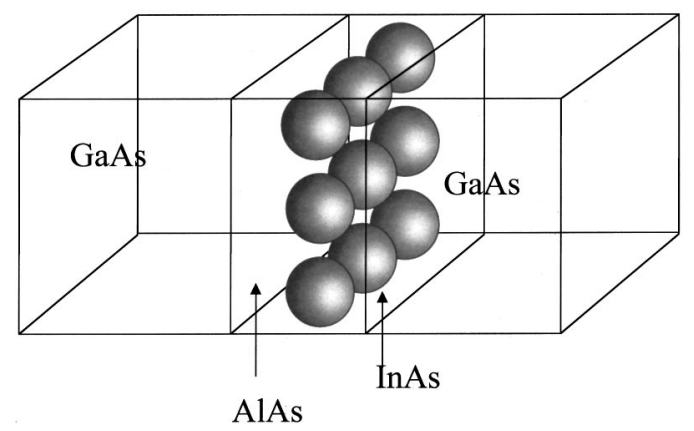

FIG. 1. Schematic diagram of a tunneling junction with a quantum dots array and two leads ( $\mathrm{GaAs}$ ) separated by a barrier of AlAs in which the InAs quantum dots array is embedded. 
which is smaller than $\Delta E$ for InAs QDs. Meanwhile,

$$
t_{i, j}=\left\{\begin{array}{l}
-t \quad \text { for nearest-neighbor } i, j \\
0 \quad \text { otherwise }
\end{array} .\right.
$$

Even though electrons in the quantum dots should experience a long range Coulomb potential, a numerical simulation of the QDs sysmtem with weak coupling shows where the wave function of the ground state of QDs is localized, and implies that the onsite Coulomb potential is a reasonable approximation as long as $t$ is much less than $U$. This localized ground state wave function of QDs is the result of high barrier potential created at interface of heterostructures. In this letter, we restrict our study to the weak coupling case.

For QDs with anisotropic shape (e.g., pyramid- or lenslike), the tunneling current can be different for forward and backward biases. This, in principle, can be modeled by a bias-dependent coupling strength between QDs and the leads in the Hamiltonian of Eq. (1). In this letter, we ignore such a dependence. In addition, we ignore band bending, roughness of interface, and inelastic scattering due to electron-phonon interaction.

Using Keldysh's Green function ${ }^{12,13}$ method and Hubbard approximation, ${ }^{14}$ we obtain the tunneling current density in proportion to the number of quantum dots $N$ as

$$
\begin{aligned}
J= & \frac{2 e N}{h} \int_{0}^{\infty} d \epsilon_{z} \int d \mathbf{k}_{\|}\left\{f_{L}\left[\epsilon(\mathbf{k})-\mu_{L}\right]-f_{R}\left[\epsilon(\mathbf{k})-\mu_{R}\right]\right\} \\
& \cdot \frac{\Gamma_{L}\left(\mathbf{k}_{\|}, \epsilon_{z}\right) \Gamma_{R}\left(\mathbf{k}_{\|}, \epsilon_{z}\right)}{\Gamma_{L}\left(\mathbf{k}_{\|}, \epsilon_{z}\right)+\Gamma_{R}\left(\mathbf{k}_{\|}, \epsilon_{z}\right)} \operatorname{Im} G_{\sigma}^{r}\left[\epsilon(\mathbf{k}), E\left(\mathbf{k}_{\|}\right)\right],
\end{aligned}
$$

where

$$
\begin{aligned}
G_{\sigma}^{r} & {\left[\varepsilon(\mathbf{k}), E\left(\mathbf{k}_{\|}\right)\right] } \\
& =\frac{\varepsilon(\mathbf{k})-U\left(1-\left\langle n_{-\sigma}\right\rangle\right)}{\varepsilon(\mathbf{k})[\varepsilon(\mathbf{k})-U]-\alpha(\mathbf{k})\left[\varepsilon(\mathbf{k})-U\left(1-\left\langle n_{-\sigma}\right\rangle\right)\right]},
\end{aligned}
$$

and

$$
\begin{aligned}
\left\langle n_{\sigma}\right\rangle= & \left\langle n_{-\sigma}\right\rangle \\
= & \frac{1}{\pi} \int_{-\infty}^{\infty} \frac{\Gamma_{L}(\omega) f_{L}\left(\omega-\mu_{L}\right)+\Gamma_{R}(\omega) f_{R}\left(\omega-\mu_{R}\right)}{\Gamma_{L}(\omega)+\Gamma_{R}(\omega)} \\
& \times \operatorname{Im} G_{\sigma}^{r}(\omega, 0) d \omega .
\end{aligned}
$$

In Eq. (2) $f_{L}\left[\epsilon(\mathbf{k})-\mu_{L}\right]$ and $f_{R}\left[\epsilon(\mathbf{k})-\mu_{R}\right]$ are the Fermi distribution function of the left and right lead, respectively. The chemical potential difference between two leads is the applied bias $\mu_{L}-\mu_{R}=\mathrm{eV}$. The kinetic energy term of the Fermi distribution functions is given by $\epsilon(\mathbf{k})$ $=\hbar^{2} k^{2} /\left(2 m_{G}^{*}\right)$, where $k$ is the wave number in the leads, and $m_{G}^{*}$ is the effective mass. In Eq. (3) $\varepsilon(\mathbf{k})=\epsilon(\mathbf{k})-E_{0}$, and $\quad \alpha(\mathbf{k})=i \Gamma-E\left(\mathbf{k}_{\|}\right), \quad$ where $\quad E\left(\mathbf{k}_{\|}\right)=2 t\left[\cos \left(k_{x} R\right)\right.$ $\left.+\cos \left(k_{y} R\right)\right]$ resulting from the interdot coupling and the symmetry in the $x-y$ plane. Here $R$ is the period length of the QDA. $\Gamma_{L}$ and $\Gamma_{R}$ denote the tunneling rate from quantum dots array to the left and right lead, respectively. Their sum is $\Gamma=\Gamma_{L}+\Gamma_{R}$. It is very difficult to fully include the tunneling rate as a function of energy and momentum, and we assume that these tunneling rates are energy and bias independent even though $\Gamma$ can be determined with a reliable method. ${ }^{10}$ According to Eqs. (3) and (4), the electron number of QDs

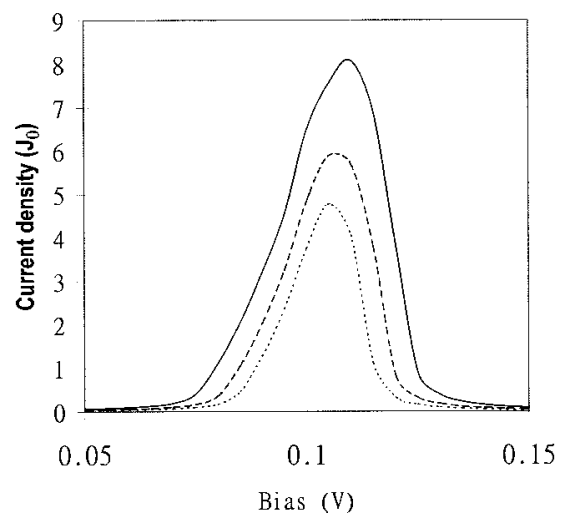

FIG. 2. Current density $J$ as a function of bias $V$ for different interdot hopping energies: $t=1.98 \mathrm{meV}$ (dotted line), $t=2.45 \mathrm{meV}$ (dashed line), and $t=3.12 \mathrm{meV}$ (solid line) for $U=0$ and zero temperature. Current density in the unit of $J_{o}=(2 e N / h)\left(2 m_{G}^{*} E_{F_{0}} / \hbar^{2}\right) \Gamma_{L} \Gamma_{R} /\left(\Gamma_{L}+\Gamma_{R}\right)$ which is used throughout this letter.

depends on the applied bias. If $\left\langle n_{\sigma}\right\rangle=0$, the spectrum of $G_{\sigma}^{r}$ has a peak around $E_{0}-E\left(\mathbf{k}_{\|}\right)$. If $\left\langle n_{\sigma}\right\rangle=1$, the spectrum of $G_{\sigma}^{r}$ shows the peak around $E_{0}+U-E\left(\mathbf{k}_{\|}\right)$.

In order to understand how Coulomb charging influences the current density, we show the current density versus bias $(J-V)$ at zero temperature for different inter-dot hopping energies in Fig. 2, where the hopping energy is defined as $t$ $=\hbar^{2} /\left(2 R^{2} m_{I}^{*}\right)$. Here $m_{I}^{*}$ is the effective mass for the $2 \mathrm{D}$ superlattice band structure of the QDA. In the weak coupling regime considered here, $m_{I}^{*}$ should be much larger than the effective mass for bulk InAs. In this study, we choose $m_{I}^{*}$ $=0.048 m_{e}$. We set the Coulomb potential $U=0, \Gamma$ $=1 \mathrm{meV}, E_{F}=50 \mathrm{meV}$, the effective mass of electron $m_{G}^{*}$ $=0.067 m_{e}$, and $E_{0}=0.1 \mathrm{eV}$. Solid, dashed, and dotted lines are for $t=3.12,2.45$, and $1.98 \mathrm{meV}$, which correspond to $R$ $=160,180$, and $200 \AA$, respectively. The current density is expressed in units of $J_{o}=(2 e N / h)\left(2 m_{G}^{*} E_{F 0} / \hbar^{2}\right) \Gamma_{L} \Gamma_{R} /\left(\Gamma_{L}\right.$ $\left.+\Gamma_{R}\right)$ throughout this letter $\left(E_{F 0}=20 \mathrm{meV}\right)$. We find that the current density is reduced as the strength of interdot hopping decreases. A decreasing $t$ implies that the volume of joint density spectrum weight $\operatorname{Im} G_{\sigma}^{r}[\varepsilon(\mathbf{k})]$ (JDSW) shrinks; therefore, current density decreases. Liu and Aers ${ }^{15}$ have calculated the tunneling current in a similar device without including the Coulomb charging.

Now we consider the Coulomb charging effect on the

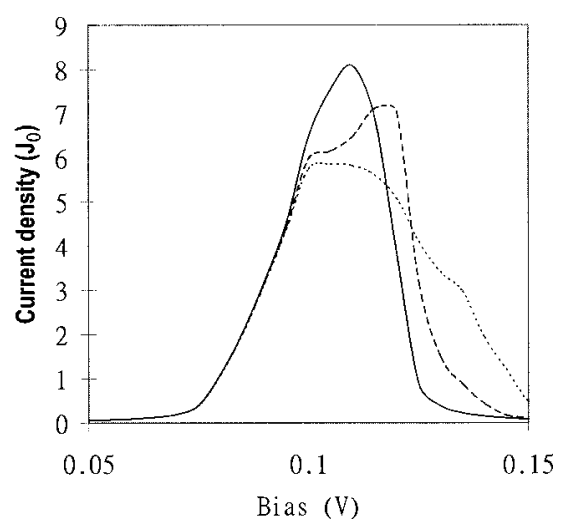

FIG. 3. Current density vs bias $V$ for different Coulomb charging energies $U$ for $t=3.12 \mathrm{meV}$. $U=0,5,10 \mathrm{meV}$, correspond, respectively, to solid line, dashed line, and dotted line. 


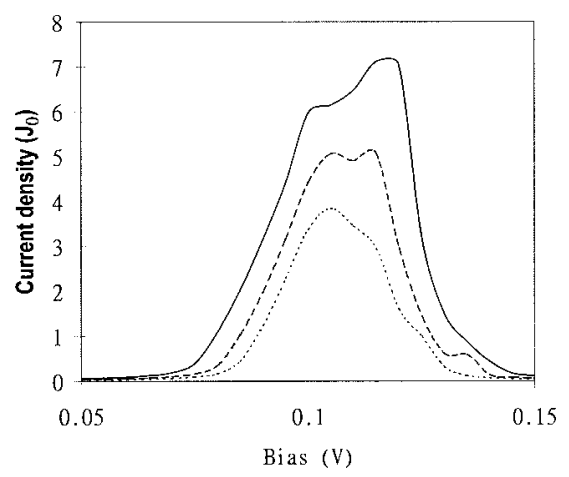

FIG. 4. Current density vs bias $V$ for different interdot hopping energies: $t=1.98 \mathrm{meV}$ (dotted line), $t=2.45 \mathrm{meV}$ (dashed line), and $t=3.12 \mathrm{meV}$ (solid line) for $U=5 \mathrm{meV}$ and zero temperature.

tunneling current for a QD array with $t=3.12 \mathrm{meV}$ for different charging energies. The results are shown in Fig. 3: solid, dashed, and dotted lines are for $U=0,5$, and $10 \mathrm{meV}$. When the Coulomb charging potential is nonzero, the band formed via the interdot coupling splits into two subbands, and JDSW consists of two peaks separated by the charging energy $U$. Consequently the current density is suppressed and pushed toward the high volatge side.

Next we show the effect of coupling strength on the current density for Coulomb charging energy $U=5 \mathrm{meV}$ in Fig. 4. The solid, dashed, and dotted lines are for $t$ $=3.12,2.45$, and $1.98 \mathrm{meV}$. For $t=3.12 \mathrm{meV}$, the curve is the same as the dashed line of Fig. 3. Similar to the behavior shown in Fig. 2, the tunneling current reduces as the coupling strength decreases however, all tunneling current spectra split into two peaks due to the effect of Coulomb blockade. We expect that as the coupling strength further increases, the electrons in the QDA will become delocalized, and the effect of Coulomb blockade will diminish. Such a study requires detailed numerical calculation which takes into account the dependence of $U$ on $t$ and the interdot Coulomb interaction, and will be considered in the future.
In conclusion, we have studied theoretically the coupling effect and Coulomb charging effect on the tunneling current density through a QD array at zero temperature. We find that the coupling of QDs lead to a two-dimensional (2D) band structure. This band will split into two subbands due to the Coulomb charging effect. The combination of the Coulomb charging effect and interdot coupling leads to pronounced structures on the tunneling current. It is found that the Coulomb charging effect can suppress the tunneling current density (or dark current). Such an effect should be taken into account in the modeling of the dark current for application in the infrared detector.

This work was supported by a Subcontract from the University of Southern California under the MURI Program, AFSOR, Contract No. F49620-98-1-0474, and also by National Science Council of Republic of China Contract Nos. NSC89-2112-M002-025 and NSC89-2212-M230-001.

${ }^{1}$ D. Goldhabar-Gordon et al., Nature (London) 391, 156 (1998).

${ }^{2}$ S. M. Cronenwett, T. H. Oosterkamp, and L. P. Kouwenhoven, Science 281, 540 (1998).

${ }^{3}$ J. H. F. Scott-Thomas, S. B. Field, M. A. Kastner, H. I. Smith, and D. A. Antoniadis, Phys. Rev. Lett. 62, 583 (1989).

${ }^{4}$ U. Meirav, M. A. Kastner, and S. J. Wind, Phys. Rev. Lett. 65, 771 (1990).

${ }^{5}$ D. C. Ralph, C. T. Black, and M. Tinkham, Phys. Rev. Lett. 78, 4087 (1997).

${ }^{6}$ P. A. Maksym and T. Chakraborty, Phys. Rev. Lett. 65, 108 (1990).

${ }^{7}$ Y. Meir, N. S. Wingreen, and P. A. Lee, Phys. Rev. Lett. 70, 2601 (1993).

${ }^{8}$ C. W. J. Beenakker, Phys. Rev. B 44, 1646 (1991).

${ }^{9}$ G. S. Solomon, J. A. Trezza, A. F. Marshall, and J. S. Harris, Phys. Rev. Lett. 76, 952 (1996).

${ }^{10}$ D. M. T. Kuo and Y. C. Chang, Phys. Rev. B 61, 11051 (2000).

${ }^{11}$ D. M. T. Kuo and Y. C. Chang, Phys. Rev. B 60, 15957 (1999).

${ }^{12}$ L. V. Keldysh, Zh. Eksp, Teor. Fiz. 47, 1515 (1964) [ Sov. Phys. JETP 20, 1018 (1965)].

${ }^{13}$ C. Caroli, R. Combescot, P. Nozieres, and D. Saint James, J. Phys. C 4, 916 (1971).

${ }^{14}$ C. Lacroix, J. Phys. F: Met. Phys. 11, 2389 (1981).

${ }^{15}$ H. C. Liu and G. C. Aers, J. Appl. Phys. 65, 4908 (1989). 
Applied Physics Letters is copyrighted by the American Institute of Physics (AIP).

Redistribution of journal material is subject to the AIP online journal license and/or AIP copyright. For more information, see http:/ojps.aip.org/aplo/aplcr.jsp

Copyright of Applied Physics Letters is the property of American Institute of Physics and its content may not be copied or emailed to multiple sites or posted to a listserv without the copyright holder's express written permission. However, users may print, download, or email articles for individual use. 